\title{
A New Technique for Hyperspectral Compressive Sensing Using Spectral Unmixing
}

\author{
Gabriel Martin ${ }^{a}$, Jose M. Bioucas $\operatorname{Dias}^{b}$ and Antonio J. Plaza ${ }^{a}$ \\ ${ }^{a}$ Hyperspectral Computing Laboratory, University of Extremadura, \\ Avda. de la Universidad s/n, 10003 Cáceres, Spain \\ E-mail: gamahefpi, aplaza@unex.es \\ ${ }^{b}$ Instituto de Telecomunicações, Instituto Superior Técnico \\ Torre Norte, Piso 10, Av. Rovisco Pais, 1049-001 Lisbon, PORTUGAL \\ E-mail: bioucas@lx.it.pt
}

\begin{abstract}
In hyperspectral imaging, the instruments measure the light reflected by the Earth surface in hundreds or thousands of spectral bands, generating huge amounts of data that must be effectively processed. The real-time requirements of some applications demand large bandwidths between the sensor and the ground stations. In order to simplify the hardware and software requirements of the hyperspectral acquisition systems, we develop a compressive sensing (CS) based technique for hyperspectral image reconstruction. CS is applicable when the data is compressible (or sparse) in a given basis or frame. This is usually the case with hyperspectral images as a consequence of its high correlation. The hyperspectral images which are compressible can be recovered from a number of measurements much smaller than the size of the original data. This compressed version of the data can then be sent to a ground station that will recover the original image by running a reconstruction algorithm. Specifically, in this work we elaborate on a previously introduced hyperspectral coded aperture (HYCA) algorithm. The performance of HYCA relies on the tuning of a regularization parameter, which is a time consuming task. Herein, we introduce a constrained formulation of HYCA, termed constrained HYCA (C-HYCA), which does not depend on any regularization parameter. C-HYCA optimization is solved with the C-SALSA alternating direction method of multipliers. In a series of experiments with simulated and real data we show that C-HYCA performance is similar to that of HYCA obtained with the best regularization parameter setting.
\end{abstract}

Keywords: Hyperspectral image analysis, compressive sensing, spectral unmixing, C-SALSA, alternating direction method of multipliers.

\section{INTRODUCTION}

Hyperspectral imaging allows an imaging spectrometer to collect hundreds of bands (at different wavelength channels) for the same area on the surface of the Earth. ${ }^{1}$ For instance, the NASA Jet Propulsion Laboratory's Airborne Visible Infra-Red Imaging Spectrometer (AVIRIS) covers the wavelength region from 0.4 to 2.5 microns using 224 spectral channels, at nominal spectral resolution of 10 nanometers. ${ }^{2}$ The resulting multidimensional data cube typically comprises several gigabytes per flight. Due to the extremely large volumes of data collected by imaging spectrometers, hyperspectral data compression has received considerable interest in recent years. ${ }^{3,4}$ These data are usually collected by a satellite or an airborne instrument and sent to a ground station on Earth for subsequent processing. Usually the bandwidth of the link connection between the satellite/airborne platform and the ground station is reduced, which limits the amount of data that can be transmitted. As a result, there is a clear need for (either lossless or lossy) hyperspectral data compression techniques that can be applied onboard the imaging instrument. ${ }^{5-7}$

In addition to extremely large dimensionality, another problem in the analysis of hyperspectral data is the presence of mixed pixels, ${ }^{8,9}$ which result from insufficient spatial resolution or due to the presence of the mixture phenomenon at different scales. Mixed pixels can also result when distinct materials are combined into a homogeneous or intimate mixture. The spectra of the individual materials which forms the mixed pixel are often called endmembers in the hyperspectral imaging literature. ${ }^{9,10}$ Linear spectral unmixing is one of the more 
simple and widely used approaches for characterizing mixed pixels in hyperspectral data. ${ }^{11}$ Let us assume that $\mathbf{Y} \in \mathbb{R}^{l \times n}$ is a hyperspectral image with $l$ bands and $n$ pixels. Under the linear mixture assumption, ${ }^{9}$ the the hyperspectral is modeled as

$$
\mathbf{Y}=\mathbf{M A}+\mathbf{N}
$$

where $\mathbf{M} \in \mathbb{R}^{l \times p}$ is a matrix containing in its columns $p$ spectral signatures of the pure materials, termed endmembers, and $\mathbf{A} \in \mathbb{R}^{p \times n}$ contains the abundance fractions associated to each endmember in each pixel of the scene. Finally, $\mathbf{N} \in \mathbb{R}^{l \times n}$ is a matrix representing the noise introduced in the model by the acquisition process. Over the last years, several techniques have been proposed for identifying the endmembers in $\mathbf{M}$ and the abundances in $\mathbf{A} .^{9,11}$ As a result, spectral unmixing has become an active research topic in recent years.

\subsection{Contribution}

In this paper, we develop a constrained version of the hyperspectral coded aperture (HYCA) algorithm introduced in, ${ }^{12}$ called constrained HYCA (C-HYCA). The new version does not depend on any regularization parameter. C-HYCA, developed under the CS framework for hyperspectral images, combines ideas of spectral unmixing ${ }^{9}$ and of compressive sensing. ?,13 As in HYCA, C-HYCA takes advantage of two main properties of hyperspectral data, namely the high spatial correlation of abundance fraction images and the low number of endmembers to explain the observed data. The former property is exploited by minimizing the total variation $(\mathrm{TV})^{14}$ of the reconstructed images of abundance fractions and the latter property is exploited by formulating the reconstruction problem with respect to abundance fraction $\mathbf{A}$, which has much lower dimension than the original data.

\subsection{Related work}

The application of compressive sensing to hyperspectral images is attracting growing attentention, both in terms of hardware and signal processing algorithms. ${ }^{15-19}$ Works ${ }^{17,18}$ have similarities with ours in the sense that they also exploit the linear mixing model and use TV regularization to recover the abundance images. They diverge, however, from our approach in many respects. Whereas our measurement matrix acts on the spectral domain, the method ${ }^{17}$ acts on the spatial domain. This has strong implications in the reconstruction algorithm and in the quality of the reconstructions, as seen below. In the case of work ${ }^{18}$ the proposed CS scheme is designed to reconstruct the original data from a single CASSI snapshot (see ${ }^{15,16}$ for details of the CASSI systems). The inference problem is a highly underdetermined and ill-posed inverse problem. Aiming at improving the conditioning of that inverse problem, the paper ${ }^{18}$ adopts the linear mixing model and proposes a joint segmentation and reconstruction of the original dual disperser CASSI measurements described in. ${ }^{15}$ In addition, the mixing matrix containing the signatures of the spectral endmembers is also estimated. In, ${ }^{19}$ the hyperspectral data cube is reconstructed by minimizing a convex functional which penalizes both the trace norm and the sum TV norms of the all image bands. These two regularizers promote, respectively, low-rank and piecewise smoothness on the reconstructed data cube. The the measurement matrix acts independently over the spatial dimension.

\subsection{Paper Organization}

The remainder of the paper is organized as follows. Section 2 describes the proposed methodology. Section 3 describes the experimental results, conducted in this work using both synthetic and real hyperspectral data. Section 3.2 concludes the paper with some remarks and hints at plausible future research lines.

\section{DESCRIPTION OF THE METHOD}

In CS the data is compressed in the acquisition process. In the case of hyperspectral imaging the compressed image is then sent to Earth. Later the original image is recovered by taking advantage hyperspectral image properties mentioned in 1.1. In this paper, we work under the assumption that we have the original image, because we need to estimate the endmembers. We note that the application of CS techniques still make sense in this scenario: the sensor acquires the complete image cube and then run a light endmember identification algorithm such as $\mathrm{VCA}^{20}$ and computes the CS measurements. It should be noted that many current efforts are intended towards the implementation (in real-time) of endmember extraction algorithms onboard the imaging instrument. ${ }^{9}$ These efforts support the operability of our proposed approach. 
The algorithm can be briefly summarized as follows. First, we partition the original hyperspectral data cube $\mathbf{Y}$ in the spatial domain into contiguous squares of size $m=w s \times w s$. Let's order the pixels in a window from 1 to $m=w_{s}^{2}$. For all windows collect the spectral vectors in the $i$ th position in matrix $\mathbf{Y}_{i}$ (one per column). As a result, depending on the size $w s$ of the window, we obtain collection of $m$ sub-sampled versions of the original image. We assume that the original data is partitioned as $\mathbf{Y}:=\left[\mathbf{Y}_{1}, \mathbf{Y}_{2}, \cdots, \mathbf{Y}_{m}\right]$.

In order to compress the original hyperspectral image, we apply a linear operator $\mathbf{H}$ to $\mathbf{Y}$ obtaining $\mathbf{Z}=$ $\mathbf{H}(\mathbf{Y}) \in \mathbb{R}^{q \times n}$ as follows:

$$
\mathbf{Z}=\mathbf{H}(\mathbf{Y}):=\left[\mathbf{H}_{1} \mathbf{Y}_{1}, \cdots, \mathbf{H}_{m} \mathbf{Y}_{m}\right],
$$

where $\mathbf{H}_{1}, \mathbf{H}_{2}, \ldots, \mathbf{H}_{m} \in \mathbb{R}^{q \times l}$ are i.i.d. Gaussian random matrices. We have then $q$ measurements per pixel. thus achieving a compression ratio of $l / q$.

Let us now define the linear operator $\mathbf{K}(\mathbf{A}):=\mathbf{H}(\mathbf{M A}) . \operatorname{In}^{12}$ we infer $\mathbf{A}$ by solving the convex optimization

$$
\min _{\mathbf{A} \geq 0} \frac{1}{2}\|\mathbf{Z}-\mathbf{K}(\mathbf{A})\|_{F}^{2}+\lambda_{T V} \operatorname{TV}(\mathbf{A}),
$$

where $\|\mathbf{X}\|_{F}:=\sqrt{\operatorname{tr}\left(\mathbf{X X}^{T}\right)}$ is the Frobenius norm of $\mathbf{X}$ and $\operatorname{TV}(\mathbf{A}):=\sum_{i=1}^{p} \operatorname{TV}\left(\mathbf{A}_{i}\right)$ is the sum of non-isotropic $\mathrm{TV}^{14}$ of the abundance images $\mathbf{A}_{i}=\mathbf{A}_{i,:}$, for $i=1, \ldots, p$. In (3), the first term measures the data misfit and the second term promotes piecewise smooth abundance images. From $\widehat{\mathbf{A}}$, the estimate of $\mathbf{A}$, we infer the original hyperspectral data set by computing $\mathbf{M} \widehat{\mathbf{A}}$.

Under the linear mixing model (1), the hyperspectral vectors belong to a subspace of dimension $p$. In this case, and if $p \ll l$, what is very often the case, taking CS measurements on the spectral domain is perhaps the best strategy. To shed light into this issue, lets assume that the number of measurements per pixel, $q$, is no smaller than the number of endmembers, $p$, and matrices $\mathbf{M}$ and $\mathbf{H}_{1}, \mathbf{H}_{2}, \ldots, \mathbf{H}_{m}$ are full rank. Then the system $\mathbf{Y}=\mathbf{K}(\mathbf{A})$ is determined if $q=p$ or overdetermined if $q>p)$, rendering a recovering problem much simpler then the usual underdetermined systems we have in CS. Of course, we are interested in pushing the compression rate to the limits, and thus, we are interested in the underdetermined measurement regime, corresponding to $q<p$.

\subsection{Constrained Formulation}

To circumvent the need to tune the regularization parameter $\lambda_{T V}$ in (3), we herein infer $\mathbf{A}$ by minimizing the following constrained version thereof:

$$
\min _{\mathbf{A} \geq 0} \operatorname{TV}(\mathbf{A}) \quad \text { subject to: } \quad\|\mathbf{Z}-\mathbf{K}(\mathbf{A})\|_{F}^{2} \leq \sigma,
$$

where $\sigma$ is a scalar value linked to the noise statistics.

To solve the problem (4), we follow closely the C-SALSA methodology introduced in. ${ }^{21}$ The core idea is to introduce a set of new variables per term of the objective function and then use the alternating direction method of multipliers $(\mathrm{ADMM})^{22}$ to solve the resulting convex constrained optimization problem. By a careful choice of the new variables, the initial problem is converted into a sequence of much simpler problems. With this in mind, let's define the linear operator $\mathbf{L}(\mathbf{A}) \equiv\left[\mathbf{L}_{h}(A) \mathbf{L}_{v}(A)\right]$ where $\mathbf{L}_{h}, \mathbf{L}_{h}: \mathbb{R}^{p n} \rightarrow \mathbb{R}^{p n}$ are linear operators computing, respectively, the horizontal and vertical differences of each band of $\mathbf{A}$ (we are assuming periodic boundaries). Thus, the non-isotropic TV regularizer can be written as $\operatorname{TV}(\mathbf{A})=\|\mathbf{L}(\mathbf{A})\|_{1,1}$, where $\|\mathbf{X}\|_{1,1}$ is the sum of the magnitude of the elements of $\mathbf{X}$. Furthermore, define $\iota_{B(\epsilon)}$ as the indicator on a ball of radius $\epsilon$, i.e., $\iota_{B(\epsilon)}(\mathbf{A})=0$ if $\|\mathbf{A}\|_{F} \leq \varepsilon$ and $+\infty$ otherwise. In a similar way define $\iota_{R_{+}}$as the indicator function of the orthant, i.e., $\iota_{R_{+}}(\mathbf{A})=0$ if every element of $\mathbf{A}$ is nonnegative and $+\infty$ otherwise. With these definition in place, an equivalent way of writing the optimization problem in (4) is

$$
\min _{\mathbf{A}}\|\mathbf{L}(\mathbf{A})\|_{1,1}+\iota_{B(\sigma)}(\mathbf{Z}-\mathbf{K}(\mathbf{A}))+\iota_{R+}(\mathbf{A}) .
$$




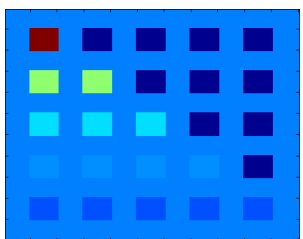

(a) Endmember \#1

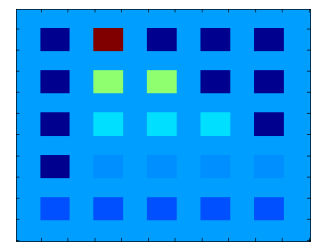

(b) Endmember \#2

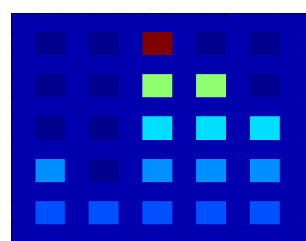

(c) Endmember \#3

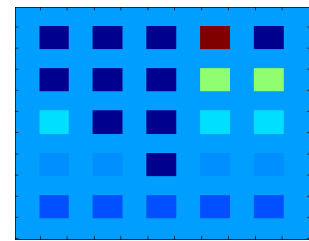

(d) Endmember \#4

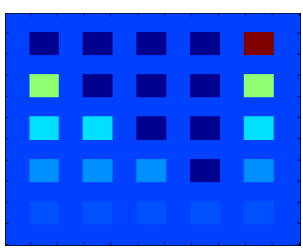

(e) Endmember \#5

Figure 1. True abundance maps of endmembers in the synthetic hyperspectral data.

Given the objective function in (5), we write the following convex constrained equivalent formulation:

$$
\begin{array}{ll}
\min _{\mathbf{A}, \mathbf{V}_{1}, \mathbf{V}_{2}, \mathbf{V}_{3}, \mathbf{V}_{4}} & \left\|\mathbf{V}_{1}\right\|_{1}+\iota_{B(\sigma)}(\mathbf{V} \mathbf{2})+\iota_{R+}\left(\mathbf{V}_{4}\right) \\
\text { subject to: } & \mathbf{L}(\mathbf{A})=\mathbf{V}_{1} \\
& \mathbf{A}=\mathbf{V}_{2} \\
& \mathbf{Z}-\mathbf{K}\left(\mathbf{V}_{2}\right)=\mathbf{V}_{3} \\
& \mathbf{A}=\mathbf{V}_{4}
\end{array}
$$

which we solve via ADMM as described in. ${ }^{23}$ The minimization of the augmented Lagrangian ( $\mathrm{see}^{23}$ for details) with respect to $\mathbf{A}$ is a quadratic problem with a block cyclic system matrix, thus effectively solved in the Fourier domain. The minimization of the augmented Lagrangian with respect to $\mathbf{V}=\left(\mathbf{V}_{1}, \mathbf{V}_{2}, \mathbf{V}_{3}, \mathbf{V}_{4}\right)$ is decoupled with respect to $\mathbf{V}_{1},\left(\mathbf{V}_{2}, \mathbf{V}_{3}\right)$, and $\mathbf{V}_{4}$. The minimization with respect to $\mathbf{V}_{1}$ is a componentwise soft-threshold and the the minimization with respect to $\mathbf{V}_{4}$ is a componentwise projection on the first orthant. The minimization with respect to $\left(\mathbf{V}_{2}, \mathbf{V}_{3}\right)$ is carried out by a block minimization with respect to $\mathbf{V}_{2}$ and to $\mathbf{V}_{3}$. The minimization with respect to $\mathbf{V}_{2}$ is a quadratic problem involving the inverse of the operator (I $\left.+\mathbf{K}\right)$ (I stands for identity operator) which, given the structure of the linear operator $\mathbf{H}$, can be precomputed. Finally, the minimization with respect to $\mathbf{V}_{3}$ is a projection on a ball of radius $\sigma$.

\section{EXPERIMENTAL RESULTS}

In this section, we conduct a series of experiments using HYCA and C-HYCA on real and simulated data.

\subsection{Synthetic data}

The synthetic dataset used in this experiments were generated from spectral signatures randomly selected from the United States Geological Survey (USGS) *. The simulated images consist of a set of $5 \times 5$ squares of $10 \times 10$ pixels each one, for a total size of $110 \times 110$ pixels. The first row of squares contains the endmembers, the second row contains mixtures of two endmembers, the third row contains mixtures of three endmembers, and so on. Zero-mean Gaussian noise was added to the synthetic scenes in with signal-to-noise ratios (SNRs) defined as $\mathrm{SNR}=10 \cdot \log _{\mathbf{1 0}}\left(E\left[(\mathbf{M A})^{\mathbf{T}}(\mathbf{M A})\right] / E\left[\mathbf{N}^{\mathbf{T}} \mathbf{N}\right]\right)$ to simulate contributions from ambient and instrumental noise sources, Fig. 1 displays the ground-truth abundances maps used for generating the simulated imagery.

In order to evaluate the performance of the $\mathrm{HYCA}$ and $\mathrm{C}-\mathrm{HYCA}$, we use as performance indicator the Normalized Mean Squared Error (NMSE) of the reconstruction given by

$$
\mathrm{NMSE}=\|\mathbf{M}(\widehat{\mathbf{A}}-\mathbf{A})\|_{F}^{2} /\|\mathbf{M A}\|_{F}^{2},
$$

where $\mathbf{A}$ and $\widehat{\mathbf{A}}$ denote the original and reconstructed images, respectively. We set the window size $w s=2$ so that $m=4$ and use the ground truth mixing matrix $\mathbf{M}$.

\footnotetext{
*http://speclab.cr.usgs.gov/spectral-lib.html
} 
Table 1. Average NMSE between the original and the reconstructed dataset for different SNR values after 10 Monte-Carlo runs.

\begin{tabular}{ccccc}
\hline \hline Version & $\mathrm{SNR}=30 d b$ & $\mathrm{SNR}=50 d b$ & $\mathrm{SNR}=70 d b$ & $\mathrm{SNR}=\infty$ \\
\hline \hline HYCA & $6.56 \cdot 10^{-4}$ & $0.29 \cdot 10^{-4}$ & $0.13 \cdot 10^{-4}$ & $0.08 \cdot 10^{-4}$ \\
\hline C-HYCA & $7.26 \cdot 10^{-4}$ & $0.51 \cdot 10^{-4}$ & $0.29 \cdot 10^{-4}$ & $0.28 \cdot 10^{-4}$ \\
\hline \hline
\end{tabular}

Table 2. NMSE between the original and the reconstructed dataset over 10 Monte-Carlo runs, for both versions of the algorithm with different compressions ratios.

\begin{tabular}{ccccccc}
\hline \hline Version & $q=5$ & $q=7$ & $q=9$ & $q=11$ & $q=13$ & $q=15$ \\
\hline \hline HYCA & $4.66 \cdot 10^{-4}$ & $4.38 \cdot 10^{-4}$ & $2.50 \cdot 10^{-4}$ & $2.46 \cdot 10^{-4}$ & $1.28 \cdot 10^{-4}$ & $1.16 \cdot 10^{-4}$ \\
\hline C-HYCA & $3.84 \cdot 10^{-4}$ & $2.72 \cdot 10^{-4}$ & $2.24 \cdot 10^{-4}$ & $1.82 \cdot 10^{-4}$ & $1.74 \cdot 10^{-4}$ & $1.70 \cdot 10^{-4}$ \\
\hline \hline
\end{tabular}

In this experiment, we set $q=3$. Since the original data set has $l=224$ bands, the compression ratio is $l / q=74.67$. Table 1 shows the value of NMSE for both versions. We performed 10 Monte-Carlo runs, sampling not only the noise but also the elements of the linear operator $\mathbf{H}$. The regularization parameter $\lambda_{T V}$ of HYCA was hand tuned for optimal performance. Having in mind the linear model (1), the parameter $\sigma$ in (4) is set to $\sigma=\|\mathbf{H}(\mathbf{N})\|_{F}$.

As we can see the values of both algorithms provide similar results, althought HYCA outperforms C-HYCA in this case. This is due to the regularization parameter $\lambda_{T V}$ of HYCA was hand tunned for optimal performance. Also the $\mathbf{H}$ linear operator is randomly generated and this may cause a little bit of variability in the results from one run to other.

\subsection{Cuprite}

In this experiment, we use the well-known AVIRIS Cuprite data set, available online in reflectance units after atmospheric correction. This scene has been widely used to validate the performance of endmember extraction algorithms. The portion used in experiments corresponds to a 250 by 190 pixels subset of the sector labeled as f970619t01p02_r02_sc03a.rfl in the online data. The scene comprises 224 spectral bands between 0.4 and 2.5 $\mu \mathrm{m}$, with full width at half maximum of $10 \mathrm{~nm}$ and spatial resolution of 20 meters per pixel. Prior to the analysis, several bands were removed due to water absorption and low SNR in those bands, leaving a total of 188 reflectance channels to be used in the experiments. In our experiments, we used a window size of $w s=2$, so that $m=4$. Here, we estimated the number of endmembers with Hysime algorithm. ${ }^{20}$

The mixing matrix $\mathbf{M}$ was estimated from the original real data using the VCA algorithm. ${ }^{24}$ Due to the non-linear mixtures and outliers present in the real images the non-negativity constraint may be violated. In order to ensure that the mixing matrix encloses the whole data-set and then the non-negativity constraint is satisfied, we open the cone defined the mixing matrix $\mathbf{M}$ as follows:

$$
\mathbf{M}^{*}:=\mathbf{M}+\Delta \cdot(\mathbf{M}-\overline{\mathbf{M}}),
$$

where $\Delta$ is a scalar that defines how much the cone is opened and $\overline{\mathbf{M}}$ is a matrix containing the mean spectrum of the endmembers. By choosing a value of $\Delta$ large enough, then all observed spectral vectors are inside the cone implying that $\mathbf{A} \geq 0$. In the current data set, $\Delta=6$ ensures this constraint. Notice that $\operatorname{span}\left(\mathbf{M}^{*}\right)=\operatorname{span}(\mathbf{M})$ and then we easily recover the estimated abundance matrix with respect to the mixing matrix $\mathbf{M}$ by computing $\widehat{\mathbf{A}}=\mathbf{M}^{\sharp} \mathbf{M}^{*} \widehat{\mathbf{A}}^{*}$, where $\widehat{\mathbf{A}}^{*}$ is the solution of (6) with respect to $\mathbf{M}^{*}$ and $\mathbf{M}^{\sharp}$ is the pseudo inverse of $\mathbf{M}$.

In order to evaluate the performance of HYCA and C-HYCA with the real dataset, we perform experiments with the compression ratios $224 / q$ with $q=5,7,11,13,15$. In all cases we used a window size of $w s=2$, so that $m=4$.

In the Table 2 we show the value of NMSE over 10 Monte-Carlo runs for the both versions of the algorithm for different compressions ratios over the Cuprite dataset. The shown values are quite similar.

Fig. 2 shows the reconstructed and the original signatures with highest, mean and lowest error for the CHYCA algorithm for different compression ratios. In this figure, we can see that even in the worse case although 


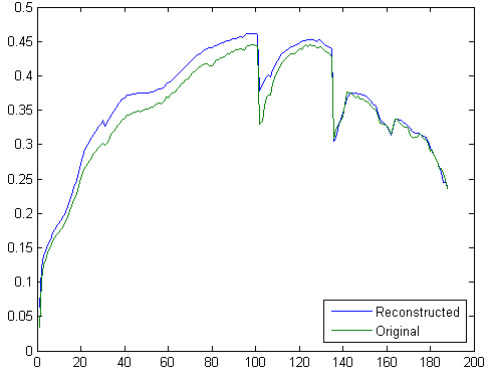

(a) $q=5$, worse case

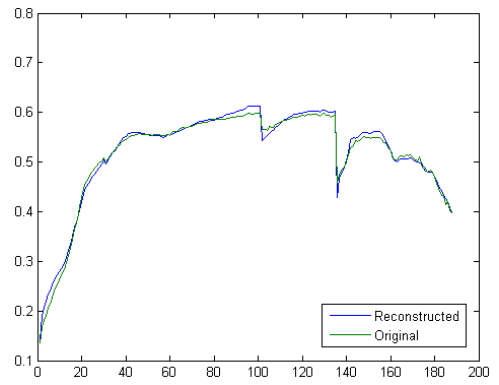

(d) $q=15$, worse case

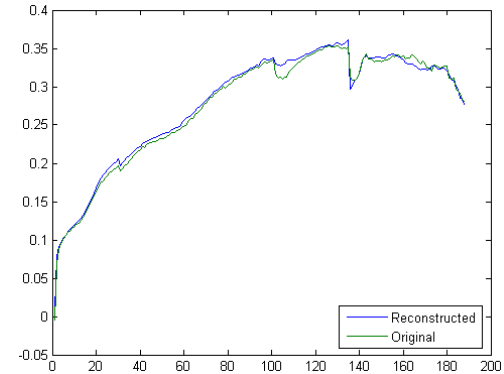

(b) $q=5$, mean case

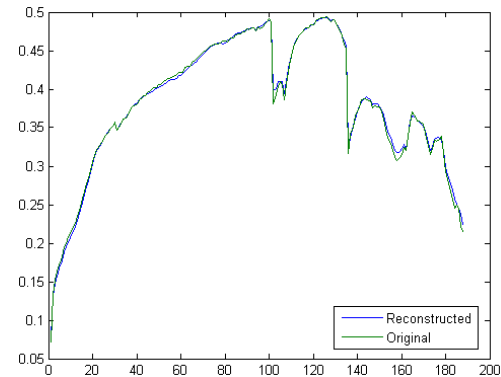

(e) $q=15$, mean case

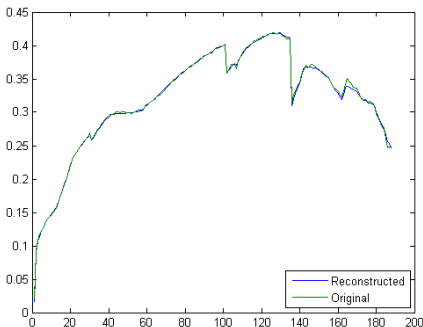

(c) $q=5$, best case

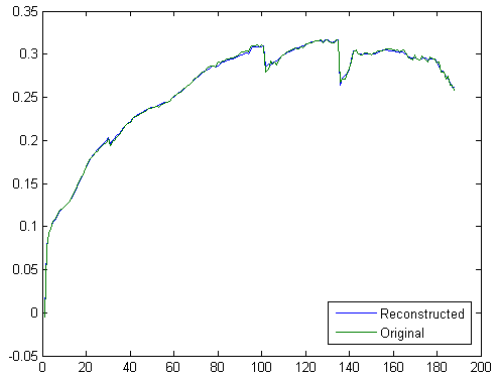

(f) $q=15$, best case

Figure 2. Worse (a), mean (b) and best (c) reconstructed pixel in the Cuprite scene for values of $q=5$ and the same in the case of $\mathrm{q}=15(\mathrm{~d}, \mathrm{e}, \mathrm{f})$.

Table 3. NMSE between the original and the reconstructed Cuprite dataset over 10 Monte-Carlo runs and values of ws $=$ $[4,6,8]$ with a compression ratio of $2334 / q$ with $q=5$.

\begin{tabular}{cccc}
\hline \hline Version & $w s=4$ & $w s=6$ & $w s=8$ \\
\hline \hline C-HYCA & $2.95 \cdot 10^{-4}$ & $3.01 \cdot 10^{-4}$ & $3.01 \cdot 10^{-4}$ \\
\hline \hline
\end{tabular}

there is a scale error between the original and the reconstructed signature, the reconstructed pixel preserves the shape of the original pixel, which means that the features of the signature are preserved. In the average case and the better case the reconstructed and the original pixel are extremely similar.

Fig. 3 shows the NMSE between the original and the reconstructed images of Cuprite for different compression ratios. Note that the scale of this figures is between 0 and $2 \cdot 10^{-3}$. Most of errors are in the transitions areas between different land-cover classes. This was expected as the TV regularizer promotes smoothness in the smooth regions. Furthermore, we can see that the most of pixels have a very low error, which means that the reconstructed and the original spectrum will be very similar in most of the cases. Note that the spectra showed in Fig. 2 correspond to the worse case, situation in which the error is much higher than the errors of the large majority of the pixels in the image.

Table 3 shows values of NMSE for the Cuprite dataset for different windows sizes $w s=[4,6,8]$ and the compression ratio of $l / q$ with $q=5$. As we can see the results are very similar for the different windows sizes. They can, however, improve a little bit by choosing an adequate window size; in this case the optimum window size of $w s=4$.

Finally in order to conclude this section, the Table 4 shows the execution time of the reconstruction algorithms

Table 4. HYCA and C-HYCA execution times for the reconstruction of Cuprite scene with 48000 pixels running 200 iterations in a INTEL CORE i7 920 CPU AT $2.67 \mathrm{GHz}$ with 4 GB of RAM

\begin{tabular}{ccc}
\hline \hline Version & Time (secs) & Time/\#pixels \\
\hline \hline HYCA & 319.78 & $6.662 \cdot 10^{-3}$ \\
\hline C-HYCA & 303.78 & $6.633 \cdot 10^{-3}$ \\
\hline \hline
\end{tabular}




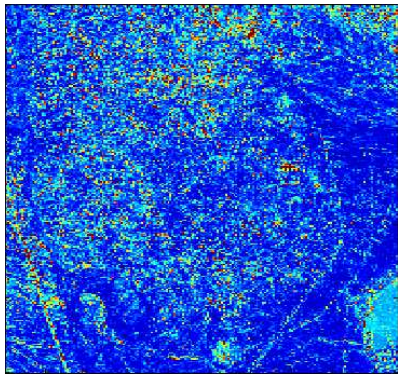

(a) $q=5$

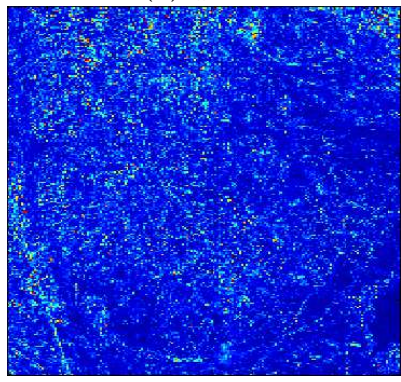

(d) $q=11$

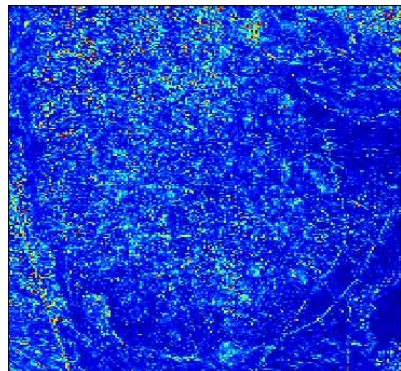

(b) $q=7$

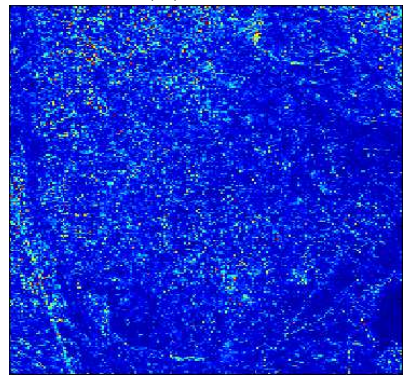

(e) $q=13$

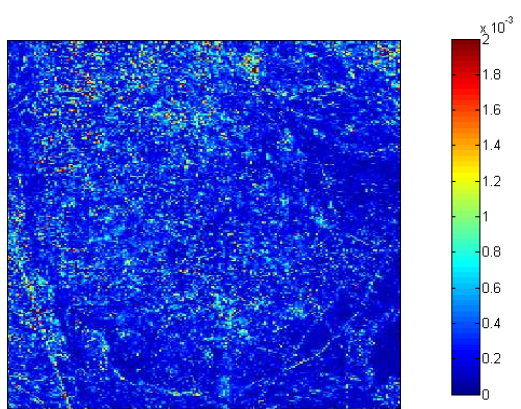

(c) $q=9$

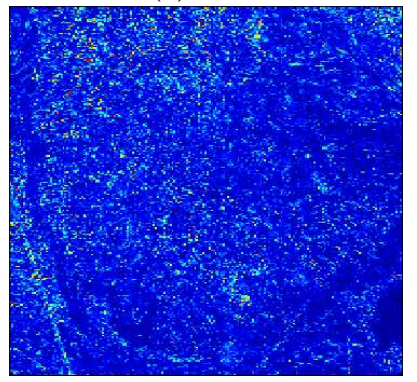

(f) $q=15$

Figure 3. NMSE between the original and the reconstructed Cuprite dataset for different compression ratios (a-f)

for the real Cuprite dataset running the algorithms during 200 iterations. This experiment was performed in a desktop computer INTEL CORE i7 $920 \mathrm{CPU}$ AT $2.67 \mathrm{GHz}$ with 4 GB of RAM. The table shows that both algorithms spend a similar time, although C-HYCA outperforms in a few seconds the HYCA algorithm. However these times have been obtained with a serial implementation, these algorithms can be implemented in high computing architectures such as clusters or GPUs which could outperform drastically the execution time.

We have introduced a Compressive Sensing algorithm called Constrained Hyperspectral Coded Aperture (CHYCA). This new algorithm is an elaboration of our perviously introduced HYCA algorithm, which depends on the tuning of a regularization parameter controlling the relative weight between the TV regularizer and the data term. Contrarily to HYCA, C-HYCA does not depend on any regularization parameter.

HYCA framework takes advantage of two main properties of hyperspectral data, namely the high spatial correlation of abundance fraction images and the low number of endmembers to explain the observed data. The former property is exploited by minimizing the total variation (TV) of the reconstructed images of abundance fractions and the latter property is exploited by formulating the reconstruction problem with respect to abundance fractions, which have much lower dimension than the original data. C-HYCA optimization problem is solved, from the numerical point of view, using the C-SALSA framework, which exploits the alternating direction method of multipliers to convert a difficult convex problem into a cyclic sequence of much simpler convex subproblems. When comparing both algorithms (HYCA and C-HYCA), it is remarkable that the quality of the reconstructions obtained for compression rations ranging between 50 and 100 is always very high in both cases. However, CHYCA offers advantages since it does not depend on the regularization parameter.

In the future we are planning on performing an estimation of the signal subspace in order to reduce data dimensionality prior to the application of the proposed method. We are also planning on developing additional strategies in order to cope with outliers in the data.

\section{REFERENCES}

1. A. F. H. Goetz, G. Vane, J. E. Solomon, and B. N. Rock, "Imaging spectrometry for Earth remote sensing," Science 228, pp. 1147-1153, 1985. 
2. R. O. Green, M. L. Eastwood, C. M. Sarture, T. G. Chrien, M. Aronsson, B. J. Chippendale, J. A. Faust, B. E. Pavri, C. J. Chovit, M. Solis, et al., "Imaging spectroscopy and the airborne visible/infrared imaging spectrometer (AVIRIS)," Remote Sensing of Environment 65(3), pp. 227-248, 1998.

3. G. Motta, F. Rizzo, and J. A. Storer, Hyperspectral data compression, Berlin: Springer, 2006.

4. B. Huang, Satellite data compression, Berlin: Springer, 2011.

5. Q. Du and J. E. Fowler, "Low-complexity principal component analysis for hyperspectral image compression," International Journal of High Performance Computing Applications 22, pp. 273-286, 2009.

6. C. Song, Y. Li, and B. Huang, "A GPU-accelerated wavelet decompression system with SPIHT and ReedSolomon decoding for satellite images," IEEE Journal of Selected Topics in Applied Earth Observations and Remote Sensing 4(3), 2011.

7. S.-C. Wei and B. Huang, "GPU acceleration of predictive partitioned vector quantization for ultraspectral sounder data compression," IEEE Journal of Selected Topics in Applied Earth Observations and Remote Sensing 4(3), 2011.

8. N. Keshava and J. F. Mustard, "Spectral unmixing," IEEE Signal Processing Magazine 19(1), pp. 44-57, 2002.

9. N. D. M. P. Q. D. P. G. J. M. Bioucas-Dias, A. Plaza and J. Chanussot, "Hyperspectral unmixing overview: geometrical, statistical, and sparse regression-based approaches," IEEE Journal of Selected Topics in Applied Earth Observations and Remote Sensing 5(2), pp. 354-379, 2012.

10. A. Plaza, P. Martinez, R. Perez, and J. Plaza, "A quantitative and comparative analysis of endmember extraction algorithms from hyperspectral data," IEEE Transactions on Geoscience and Remote Sensing 42(3), pp. 650-663, 2004.

11. A. Plaza, Q. Du, J. Bioucas-Dias, X. Jia, and F. Kruse, "Foreword to the special issue on spectral unmixing of remotely sensed data," IEEE Transactions on Geoscience and Remote Sensing 49(11), pp. 4103-4110, 2011.

12. J. B.-D. G. Martin and A. Plaza, "Hyperspectral coded aperture: A new algorithm for hyperspectral compressive sensing," IEEE Geoscience and Remote Sensing Symposium (IGARSS'12), (Munich, Germany), 2012.

13. D. Donoho, "Compressed sensing," IEEE Transactions on Information Theory, pp. 1289-1306, 2006.

14. L. Rudin, S. Osher, and E. Fatemi, "Nonlinear total variation based noise removal algorithms," Physica D: Nonlinear Phenomena 60(1-4), pp. 259-268, 1992.

15. M. G. R, John, D. D. Brady, R. Willett, and T. Schulz, "Single-shot compressive spectral imaging with a dual-disperser architecture," Optics Express 15(21), pp. 14013-14027, 2007.

16. A. Wagadarikar, R. John, R.-W. D., and Brady, "Single disperser design for coded aperture snapshot spectral imaging," Applied optics 47(10), pp. B44-B51, 2008.

17. C. Li, T. Sun, K. Kelly, and Y. Zhang, "A compressive sensing and unmixing scheme for hyperspectral data processing," IEEE Transactions on Image Processing 21(3), pp. 1200-1210, 2011.

18. Q. Zhang, R. Plemmons, D. Kittle, D. Brady, and S. Prasad, "Joint segmentation and reconstruction of hyperspectral data with compressed measurements," Applied Optics 50(22), pp. 4417-4435, 2011.

19. M. Golbabaee and P. Vandergheynst, "Joint segmentation and reconstruction of hyperspectral data with compressed measurements," in IEEE International Conference on Image Processing ICIP2012, 2012., 2012.

20. J. M. Bioucas-Dias and J. M. P. Nascimento, "Hyperspectral subspace identification," IEEE Transactions on Geoscience and Remote Sensing 46(8), pp. 2435-2445, 2008.

21. M. Afonso, J. Bioucas-Dias, and M. Figueiredo, "An augmented Lagrangian approach to the constrained optimization formulation of imaging inverse problems," IEEE Transactions on Image Processing 20(3), pp. $681-695,2011$.

22. J. Eckstein and D. Bertsekas, "On the Douglas-Rachford splitting method and the proximal point algorithm for maximal monotone operators," Mathematical Programming 5, pp. 293-318, 1992.

23. J. Bioucas-Dias and M. Figueiredo, "Alternating direction algorithms for constrained sparse regression: Application to hyperspectral unmixing," 2nd IEEE GRSS Workshop on Hyperspectral Image and Signal Processing-WHISPERS'2010 , (Raykjavik, Iceland), 2010.

24. J. M. P. Nascimento and J. M. Bioucas-Dias, "Vertex Component Analysis: A Fast Algorithm to Unmix Hyperspectral Data," IEEE Transactions on Geoscience and Remote Sensing 43(4), pp. 898-910, 2005. 